\title{
Oral Involvements of Hypersensitivity Reactions to ChemotherapyDrugs in Immunocompromised Child Patient: A Case Report
}

\author{
Agam Ferry Erwana*, Irna Sufiawati \\ Department of Oral Medicine, Faculty of Dentistry, Universitas Padjadjaran, Bandung 40134, Indonesia \\ *E-mail: doktergigigaul@gmail.com
}

\begin{abstract}
Background: Hypersensitivity reactions (HSRs) may occur in children with cancer during the use of almost all chemotherapeutic drugs, commonly present with mild/moderate to severe clinical patterns.

Case report: A 7-year-old, male patient, was referred from the RSUP Hasan Sadikin Pediatric Department, with the diagnosis of Acute Lymphocytic Leukemia (ALL), acute otitis media and Toxic Epidermal Necrolysis (TEN) ec susp/ metrotreksat ec susp/ leucovorine. The patient was diagnosed with oral involvement related to anemia and TEN. Lesions of reddish black crusts manifested on upper and lower lips, and multiple red macules in labial and buccal mucosas. Oral lesions showed significant improvement after administration of comprehensive treatment including corticosteroid mixed ointment, antimitotic oral suspension and multivitamin.

Discussion: The mechanisms responsible for most HSRs, including drug-related are not known, as they have generally not been evaluated. Most reactions are of the type I category in the Gell and Coombs classification, but there also are instances of types II, III, and IV reactions caused by many of the antineoplastic agents. Oral involvements in children due to hypersensitivity reactions are likely to occurred because they are more susceptible to stomatotoxic effects of chemotherapeutic agents, possibly related to a higher epithelial mitotic rate.
\end{abstract}

Conclusion: Knowledge of the different clinical presentations of chemotherapeutic agent's hypersensitivity reactions 'oral involvements can help to ensure a good treatment outcome, to improve the quality of patient care and to reduce healthcare costs.

Keywords: acute lymphocytic leukemia, chemotherapy, hypersensitivity reactions, oral involvement

\section{Introduction}

Oral Medicine is a specialty that deals with the diagnosis and medical management of the complex medical disorders involving the oral mucosa. Almost $99.9 \%$ of systemic diseases have one or more oral manifestations which are diagnosed by oral physician even before the general physician. Having early recognition and diagnosis are both important for early treatment, in order to improve patient survival and for limiting the complications of therapy. ${ }^{1,2}$

Several systemic factors are known to contribute to oral diseases or conditions. Among those are the intakes of drugs. The pathogenesis of oral adverse reactions related to intake of medications has not yet been well-understood, and the prevalence is still not known. They are, however, believed to be a relatively common phenomenon, although medication-induced oral reactions are often regarded by the health profession as trivial complaints. ${ }^{2}$
Patients receiving multiple doses of chemotherapy can become sensitized to the drugs. Thus, subsequent exposure to these agents can lead to hypersensitivity reactions (HSRs) and death. ${ }^{3}$ HSRs may occur in children with cancer, treated with chemotherapeutic drugs (especially platinum compounds, methotrexate, Lasparaginase), with mild/moderate to severe clinical patterns. ${ }^{4}$ It often requires that the provoking medication be discontinued. But this is also raising a dilemma for the caregiver, since further use could precipitate a severe, even fatal, allergic reaction on re-exposure, but alternative drugs might be poorly tolerated or much less effective compared with the preferred agent. ${ }^{3}$

Complications resulting from cancer, or secondary to cancer treatment, frequently occur in the oral cavity and are often nonspecific. The reported incidence of such complications in the literature varies considerably, but they appear to be far more common in children ${ }^{5}$.

In this paper, we try to reports oral involvements of hypersensitivity reactions in child patient with Acute 
Lymphoblastic Leukemia underwent chemotherapy, to improve the knowledge about drug-induced oral adverse effects. Eventually that will help better oral disease diagnosing process, drugs administration, improve patient compliance during drug therapy, and may influence a more rational use of drugs in immunocompromised patients.

\section{Case Report}

On June 17th, 2016, a 7-year-old, male patient, came to the Oral Medicine Department with major complaints of gingival bleeding and painful oral ulcers. The patient was referred from the RSUP Hasan Sadikin Pediatric Department with working diagnosis of ALL, acute otitis media, febrile neutropenia, chronic apical periodontitis ec gangrene radix and TEN ec susp/ metrotreksat ec susp/ leucovorine, for oral lesions management.

Around 10 days prior to his admittance to RSUP Hasan Sadikin he had chief complaints of pain in the right inner buccal and lower inner lip, on eating, talking, gargling and swallowing. The patient had history of underwent chemotherapy treatment around 2 weeks ago. Medical history also reveals that the patient suffered from acute otitis media and febrile neutropenia. History of medication from the Dermato-Venereology Department were metilprednisolon $8 \mathrm{mg}$ tablet two times a day (1-1-0), vaseline salf and dexamethasone amp $60 \mathrm{ml}$ in $100 \mathrm{cc}$ $\mathrm{NaCl}$.

The general condition of the patient looks weak, but still cooperative and communicable. In extraoral examination, no lymph nodes were palpable. The lips were dry and there are reddish black crust lesions covering lower lip almost entirely with spontaneous bleedings and reddish papules in the upper lip ranging from 13 to 21 (Figure 1). The left and right lip border shows macule lesions, paler than its surrounding tissue in color, irregular in shape, with diameter $<5 \mathrm{~mm}$. No "target" lesion was found on the skin surface of his body or extremities.

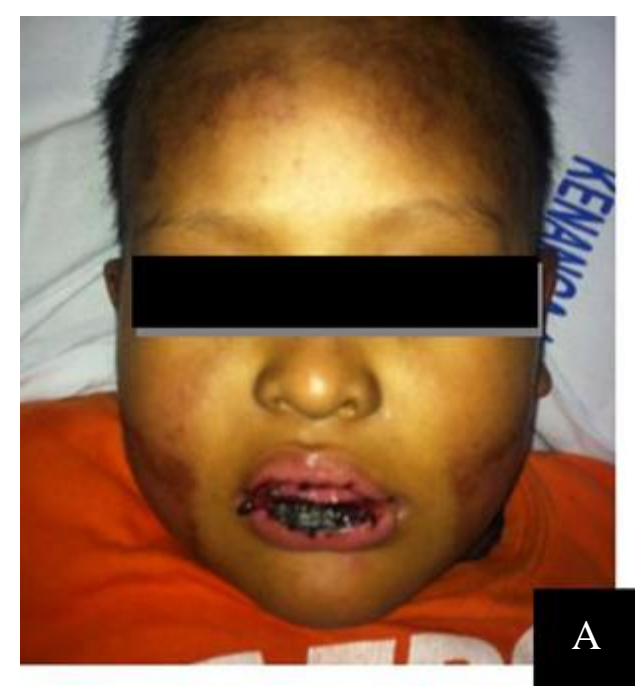

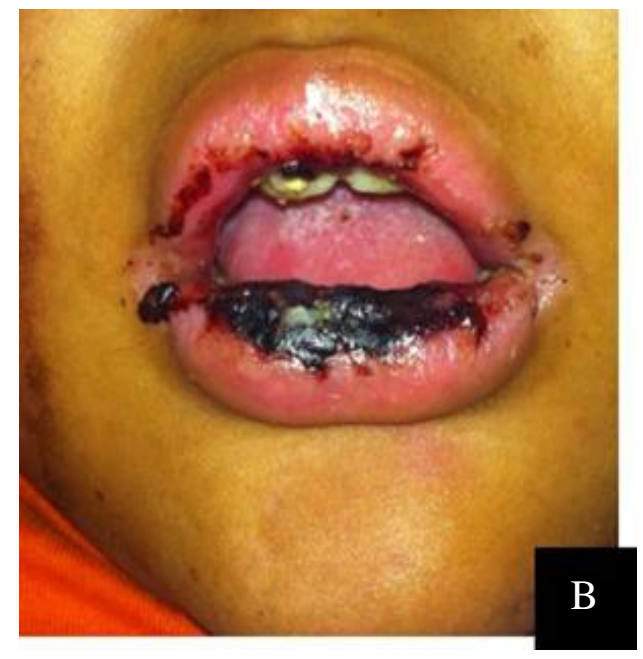

Figure 1. Extraoral view and lesions. A. Extraoral view. B. Reddish black crust lesions on lower lips and spontaneous bleedings and reddish papules in the upper lip

Intraoral examination showed multiple, erythem macule lesions, irreguler shape, with pain in labial mucosas. In buccal mucous there are also erythem macule lesions covered with multiple white plaque, $<5 \mathrm{~mm}$ in diameter and no pain involved. The tongue dorsal was covered with white plaque, leaving painful erythema base on scrapping, while in tongue ventral there is an erythem macule lesion, with no pain. Palatum was anemic. Gingival edema presented both in upper and lower jaws with plaque and calculus presents (Figure 2). These ulcers were very painful and prevented him from carrying out his daily routines.

As history and clinical features were suggestive of hypersensitivity reactions and autoimmune disorders manifestations the diagnosis of oral involvements related to anemia, oral involvements related to TEN ec. metrothrexate ec leucovorin and oral candidiasis were formed. 


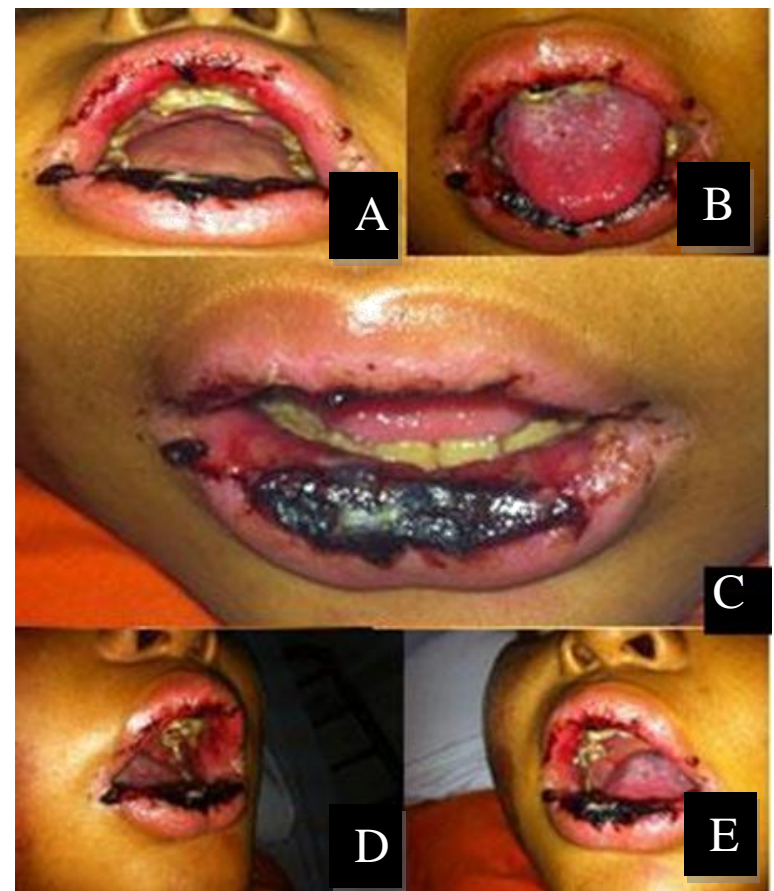

Figure 2. Intraoral lesions. A. Palatal region. B. Tonguedorsal region. C. Lower labial mucosa. D. Left buccal mucosa. E. Right buccal mucosa

Treatments for this patient were as follows: oral hygiene instructions, cie (communication, information and education) about the oral lesions, complications and treatment planning, B12 vitamins given thrice a day, folic acid once daily, nystatin oral suspension $1 \mathrm{ml} 4$ times daily, and topical dexamethasone concoction zalf. The concoction zalf were made by mixturing dexamethasone, avil, lanolin and vaseline. The treatments were scheduled for 7 days.

The child was seen 1 week later in control visit, with good resolution of symptoms. Lesions in several regions had completely cleared up after the treatment, sparse with mild resolving erythema and hyperpigmentation. Since the patient cannot open his mouth in previous visit, oral ulcers in buccal mucosas covered with necrotic pseudo membrane with no pain is now observed. (Figure 3). Treatment were planned to give Triamcinolone acetonide in orabase $0,1 \%$ applied directly on the lesions thrice daily.

\section{Discussion}

During the chemotherapy treatment for acute leukemia (AL), a patient may experience a wide variety of complications that mainly have three possible origins, namely the disease itself (leukemic infiltration), peripheral blood cell depression (because of hemorrhagic or infectious processes) and toxicity induced by chemotherapy. Patients receiving multiple doses of chemotherapy can become sensitized to the drugs, and subsequent exposure to these agents can lead to hypersensitivity reactions (HSRs) and death. ${ }^{3}$
A wide spectrum of drugs can sometimes give rise to numerous adverse oral manifestations, particularly dry mouth, taste disturbances, swelling, and/or oral mucosal ulceration.6 Hypersensitivity reactions (HSRs) may occur in patients treated with chemotherapeutic drugs (especially platinum compounds, methotrexate, Lasparaginase), with mild/moderate to severe clinical patterns ${ }^{4}$.

The oral involvements in this patient are suspected due to subsequent exposure to chemotherapeutic drugs, such as methotrexate and leucovorin, because the patient had No. complaining in his oral mucosas when the patient is not in chemotherapy cycle. Thus, whenever the patient was reexposure to those drugs, the hypersensitivity oral manifestations arisen.

The mechanisms responsible for most HSRs are not known, as they have generally not been evaluated. Most reactions are of the type I category in the Gell and Coombs classification, but there also are instances of types II, III, and IV reactions caused by many of the antineoplastic agents. Many patients can subsequently tolerate re-exposure after pretreatment with steroids and antihistamine, and slow re-administration of the drug ${ }^{1}$.

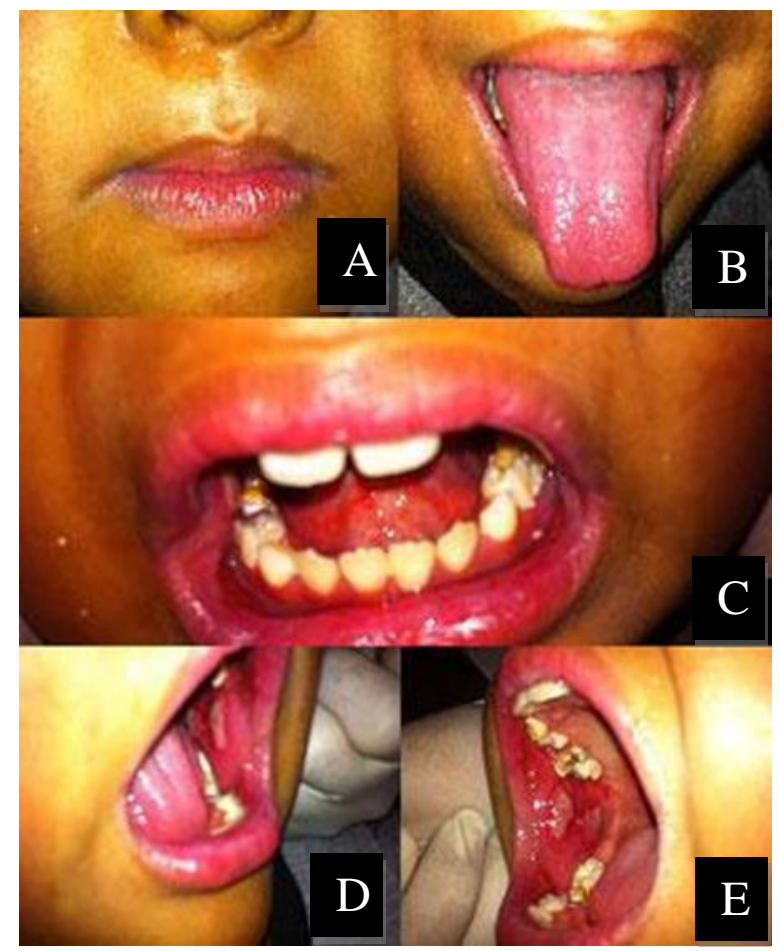

Figure 3. Intraoral lesions after 1 week control. A. Lower lip region. B. Dorsal tongue region. C. Lower labial mucosa. D. Left buccal mucosa. E. Right buccal mucosa

Direct stomatotoxic effects begin shortly after therapy is begun and usually peak at about day. ${ }^{7}$ The severity ranges from mild mouth soreness with a paucity of clinical findings to severe erosive ulcers that is accompanied by severe pain and an inability to eat or drink. ${ }^{7}$ This is consistent with the anamnesis retrieved from the patient, who had complaint while eating and talking and unable to perform his daily routines very well. 
Multiple factors influence the extent and severity of stomatotoxic effects, including the specific drug, dose, route and frequency of administration, and individual patient tolerance. Chemotherapeutic agents that are DNA cycle-specific (eg, bleomycin, 5-fluorouracil (5-FU), and methotrexate) are more stomatotoxic than those that are cell phase non-specific. Certain drugs (eg, methotrexate, etoposide) are also may be secreted into the saliva, further increasing the potential for oral manifestations of stomatotoxicity. $^{7}$

The incidence of mucosal ulceration and inflammation may reflect the use of more aggressive chemotherapeutic regimens in treating childhood malignancy during recent years. The apparent relationship between the administration of methotrexate and oral ulceration was in agreement with studies by White (1970). Not all patients who received methotrexate developed ulceration, and those who did develop ulceration did not suffer oral mucosal reactions on every occasion they received the drug. Dosages that produced severe oral reactions in some patients caused no problems in others of the same age with the same malignancy. ${ }^{5}$

Younger patients have a relatively greater risk of chemotherapy-induced hypersensitivity reactions, perhaps related to a higher epithelial mitotic rate ${ }^{8}$. But there are also other factors that may modulate the severity of stomatitis include nutritional status, the type of malignancy, the quality of oral care during treatment, pretreatment neutrophil counts, and the use of hematopoietic growth factor support during therapy. ${ }^{9}$

Toxic epidermal necrolysis (TEN) is an uncommon fulminating mucocutaneous disease associated with marked morbidity and mortality. While sometimes idiopathic, TEN is frequently associated with drug administration (Table 1). ${ }^{10}$ Intra-orally, there is widespread painful blistering and ulceration of all mucosal surfaces.

The first large study to assess the risk of developing Steven Johnson Syndrome (SJS) or TEN, distinguished between drugs usually used for short-term periods and drugs used for months or years. Specific drug hypersensitivity leads to major histocompatibility class I restricted drug presentation and is followed by an expansion of cytotoxic T-lymphocytes, leading to an infiltration of skin lesions with cytotoxic T-lymphocytes and natural killer cells. Granulysin probably is the key mediator for disseminated keratinocyte death in TEN. ${ }^{11}$

The differential diagnosis of oral involvements related to anemia, oral involvements related to TEN ec. methotrexate ec leucovorin and candidiasis were formed in this case, based on anamneses, medical history, clinical features and diagnosis from the pediatric department.
Table 1. Drug-related erythema multiforme (and StevensJohnson syndrome and toxic epidermal necrolysis)6

\begin{tabular}{lll}
\hline Drug names & Drug names & Drug names \\
\hline Allopurinol & Fluconazole & Progesterone \\
Amlodipine Arsenic & Fluorouracil & Pyrazolone- \\
Atropine Busulphan & Furosemide & derivatives \\
Carbamazepine & Gold & Quinine \\
Chloral hydrate & Griseofulvin Hydantoin & Sulindac \\
Chloramphenicol & Hydrochlorothiazide & Sulphasalazine \\
Chlorpropamide & Indapamide & Tenoxicam \\
Clindamycin & Mercury & Tetracyclines \\
Codeine & Meclofenamic-acid & Theophylline \\
Co-trimoxazole & Nifedipine & Tocainide \\
Diclofenac & Omeprazole & Tolbutamide \\
Diflunisal & Penicillin derivatives & Trimethadione \\
Digitalis & Phenolphthalein & Vancomycin \\
Diltiazem & Phenylbutazone & Verapamil \\
Ethambutol & Phenytoin & Zidovudine \\
Ethyl-alcohol & Piroxicam & \\
& & \\
\hline
\end{tabular}

Corticosteroid concoction zalf, B12 vitamins and folic acid were given for treatments of the patient to help the lesions of lip crust to heal, while nystatin was prescribed for the oral candidiasis. B12 vitamins and folic acid is important in intracells metabolism. Both of this vitamin play roles in reepithelization process. Oral involvement of TEN causing ulceration and crust of oral mucosas, therefore giving these vitamins can recovered those lesions in this case. ${ }^{11}$

It needs to take into considerations that whenever a patient comes with oral lesions, ask about their history of medications. If it seems significant then either reduce to the minimum dose required or switch to alternative regimen depending upon the severity of symptoms. Sometimes active treatment of the concerned effect may also be required. ${ }^{1}$ A multidisciplinary approach to medical consultation and appropriate referrals ensures comprehensive medical and dental management of patients.

\section{Conclusions}

Prevention and treatment of chemotherapy stomatotoxic effects, especially in children, needs special attention, since they are more susceptible to the effects 5 . The rapid progress in pharmacotherapeutic requires clinicians to constantly update their knowledge of drugs used by their patients. Attention must be paid to their toxic and unwanted effects, especially oral involvement, that in many cases maybe similar to characteristics of common diseases. $^{12}$

The majority of chemotherapeutic drug-induced oral reactions are moderate in severity. In most cases, the oral reaction will be resolved by symptomatic treatment ${ }^{13}$. In view of the significant existing dental disease at diagnosis and the subsequent very high incidence of complications in and around the oral cavity, it is clear that the dentist has a very important role in the initial assessment and ongoing care of the pediatric oncology patient. ${ }^{5}$ 


\section{References}

1. Pejcic A. Drug-Induced Oral Reactions. In: Emerging Trends in Oral Health Sciences and Dentistry [Internet]. InTech; 2015 [cited 2016 Aug 23]. Available from: http://www.intechopen.com/books/emerging-trends-in-oralhealth-sciences-and-dentistry/drug-induced-oral-reactions

2. Porter SR, Scully C. Adverse drug reactions in the mouth. Clin Dermatol. 2000;18(5):525-32.

3. Castells MC, Tennant NM, Sloane DE, Ida Hsu F, Barrett NA, Hong DI, et al. Hypersensitivity reactions to chemotherapy: Outcomes and safety of rapid desensitization in 413 cases. J Allergy Clin Immunol. 2008 ;122(3):574-80.

4. Ruggiero A, Triarico S, Trombatore G, Battista A, Dell'Acqua F, Rizzari C, et al. Incidence, clinical features and management of hypersensitivity reactions to chemotherapeutic drugs in children with cancer. Eur JClin Pharmacol. $2013 ; 69(10): 1739-46$.

5. Fayle SA, Curzon ME. Oral complications in pediatric oncology patients. Pediatr Dent. 1991;13(5):289-95.

6. Scully C, Bagan-Sebastian JV. Adverse drug reactions in the orofacial region. Crit Rev Oral Biol Med. 2004;15(4):221-40.
7. Robert S Negrin M, Jean-Francois Bedard D, Joseph A Toljanic D. Oral toxicity associated with chemotherapy [Internet]. Available from: http://cursoenarm.net/UPTODATE/contents/mobipreview.h tm?36/23/37233?source=related_link

8. Sonis ST, Sonis AL, Lieberman A. Oral complications in patients receiving treatment for malignancies other than of the head and neck. J Am Dent Assoc. 1978;97(3):468-72.

9. Barasch A, Peterson DE. Risk factors for ulcerative oral mucositis in cancer patients: Unanswered questions. OralOncology. 2003. p. 91-100.

10. Livasy CA, Kaplan AM. Ciprofloxacin-induced toxic epidermal necrolysis: a case report. Dermatology. 1997 ;195(2):173-5.

11. Deore SS, Dandekar RC, Mahajan AM, Shiledar V V. Drug Induced -Stevens Johnson Syndrome: A Case Report. 2014;2(4):84-7.

12. Moore PA, Gage TW, Hersh EV, Yagiela JA, Haas DA. Adverese drug interactions in dental practice. $\mathrm{J}$ Am DentAssoc. 1999;130(1):47-54.

13. Abdollahi M, Rahimi R, Rafdar M. Current Opinion on Drug-induced Oral Reactions: A Comprehensive Review. J Contemp Dent Pract. 2008;9(3). 